\title{
Combining Ability and Heterosis for Kernel Yield and Yield Attributes in Maize (Zea mays L.)
}

\author{
N. Sabitha*, D. Mohan Reddy, D. Lokanath Reddy, M. Hemanth Kumar, \\ P. Sudhakar and B. Ravindra Reddy
}

Department of Genetics and Plant breeding, S.V. Agricultural College, Tirupati-517502, A.P., India

*Corresponding author

\section{A B S T R A C T}

\section{Keywords}

Multi-parent hybrids, heterosis, cross-pollinated crop, biological feasibility

\section{Article Info}

\section{Accepted:}

12 May 2021

Available Online:

10 June 2021
Analysis of 45 cross combinations made in a half diallel mating and studied in Rabi, 2016-17 for yield and yield components revealed that both additive and non-additive type of gene actions with predominance of non-additive gene action was found to be important for days to $50 \%$ tasseling, days to $50 \%$ silking, plant height, SPAD chlorophyll meter reading (SCMR), cob length $(\mathrm{cm})$, cob girth $(\mathrm{cm})$, number of kernel rows per cob, number of kernels per row, 100 kernel weight and kernel yield per plant. The crosses viz., BML15×PDM1474, BML7×DFTY, and BML15×PDM1452 were considered as outstanding crosses as they have recorded significant positive heterosis and sca effects in the desirable condition for most of the yield contributing characters and hence can be recommended for commercial cultivation after extensive testing under different environments and locations. All other cross combinations involving good $\times$ poor, poor $\times$ good or poor $\times$ poor may be handled through heterosis breeding while the cross combinations with good $\times$ good combiners through recurrent selection programmes to develop high yielding hybrids and isolate improved inbred lines, respectively.

\section{Introduction}

Maize (Zea mays L.) is a highly crosspollinated crop and the scope for exploitation of hybrid vigour will depend on the direction and magnitude of heterosis, biological feasibility and the type of gene action involved. The magnitude of heterosis provides information on the extent of genetic diversity of parents involved in developing superior $F_{1}$ 's so as to exploit hybrid vigour. Single cross hybrids are the most desirable as the breeding and seed production is much easier than the multi-parent hybrids (Vasal et al., 1995). This necessitates identification of superior inbreds for further enhancing 
productivity of hybrids. Gene action studies facilitates decision making of a suitable breeding procedure for genetic improvement of various quantitative traits (Jinks and Hayman, 1963) (Reza et al.,2004 and Begum, et al., 2013). Diallel cross analysis provides the estimates of genetic parameters viz., combining ability and dominance relationship of the parents used for the first filial generation with or without reciprocals. The present investigation involving 45 cross combinations derived through diallele mating design was carried to identify the superior hybrid combinations, gene action governing different yield components and the magnitude of heterosis for yield and yield components based on combining ability studies.

\section{Materials and Methods}

Ten diverse maize inbred lines were crossed in all possible combinations without reciprocals. Inbreds and their $\mathrm{F}_{1}$ hybrids were tested in a RBD with three replications during Rabi, 2016-17at Agricultural Research Station, Perumallapalli. Each genotype was planted in a row of 5 meter length. The spacing between row to row was $60 \mathrm{~cm}$ and plant to plant was $20 \mathrm{~cm}$. A fertilizer dose of $120 \mathrm{~N}, 80 \mathrm{P}_{2} \mathrm{O}_{5}, 60$ $\mathrm{K}_{2} \mathrm{O} \mathrm{kg} / \mathrm{ha}$ was applied. All the best agronomic management practices and plant protection measures were followed in raising a healthy crop. Observations were recorded on five randomly selected plants from each plot for plant height $(\mathrm{cm})$, SPAD chlorophyll meter readings, cob length $(\mathrm{cm})$, cob girth $(\mathrm{cm})$, number of kernel rows per cob, number of kernels per row, 100 kernel weight $(\mathrm{g})$ and kernel yield per plant (g). Days to 50\% tasseling and days to $50 \%$ silking were recorded on whole plot basis. SPAD chlorophyll meter reading (SCMR) on third leaf was measured with a SPAD -502 chlorophyll meter (Minolta Co Ltd, Osaka, Japan). Analysis of variance and mean comparison of characters were done using
SAS software. Diallel cross analysis (Model I and Method IV) was followed for performing partitioning the sum of squares of genotypes in to general and specific combining ability and to estimate their effects for different cross combinations, respective standard errors and genetic components of variances using the formulae given by Griffing (1956). Heterosis expressed as per cent increase of $F_{1}$ hybrid over standard check (Standard heterosis) was computed for developmental characters, kernel yield and yield components using the formulae (Turner, 1953 and Hayes et al., 1985).

\section{Results and Discussion}

\section{Combining ability and Components of Genetic variances}

Data on partitioning of genotypic variance in to mean sum of squares of GCA and SCA and components of genetic variance were presented in Table 1.

Mean performance, sca effects and heterosis computed over standard check (DHM 117; BML2×BML6) in respect of top 20 single cross hybridsfor days to $50 \%$ tasseling, days to $50 \%$ silking, plant height $(\mathrm{cm})$, SPAD chlorophyll meter reading (SCMR), cob length $(\mathrm{cm})$, cob girth $(\mathrm{cm})$, number of kernel rows per cob, number of kernels per row, 100 kernel weight $(\mathrm{g})$ and kernel yield per plant $(\mathrm{g})$ was presented in Table 2.

Mean sum of squares due to general combining ability (GCA) and specific combining ability SCA) were significant for days to $50 \%$ tasseling $\left(7.66^{* *}\right.$ and $\left.4.11^{* *}\right)$, days to $50 \%$ silking (10.83** and 5.99**), plant height (335.63** and 296.56**), SPAD chlorophyll meter reading $(20.62 * *$ and $7.27 * *)$, cob length (5.94** and $\left.1.88^{* *}\right)$, cob girth $\left(3.01^{* *}\right.$ and $\left.1.01 * *\right)$, number of kernel rows per $\operatorname{cob}\left(3.63^{* *}\right.$ and $\left.0.74 * *\right)$, number of 
kernels per $\operatorname{row}(28.00 * *$ and $6.52 * *), 100$ kernel weight (14.71** and 4.15**) and kernel yield per plant $\left(1196.97 * *\right.$ and $\left.308.83^{* *}\right)$, respectively. The mean sum of squares of GCA includes the additive and additivexadditive genetic portion while SCA represents the non-additive genetic portion of the total variance arising largely from dominance and epistatic deviations. The significant mean sum of squares due to GCA and SCA obtained in the present investigation indicates that both additive and non-additive gene action are involved in the expression of all the characters studied. Similar findings in maize were also reported by Iqbal et al., (2007) and Hefney (2010).

The estimates of genetic components of variance computed to find out which type of gene action is more important for the above characters revealed the greater the magnitude of non-additive genetic component of variance $\left(\sigma^{2}\right.$ sca) than additive component $\left(\sigma^{2}\right.$ gca) suggesting that non-additive component is predominant for days to $50 \%$ tasseling $(3.61 * *$ and $\left.0.90^{* *}\right)$, days to $50 \%$ silking (5.33** and $1.27 * *$ ), plant height (290.90** and 35.62**), SPAD chlorophyll meter reading $(6.34 * *$ and $\left.2.46^{* *}\right)$, cob length $\left(1.48^{* *}\right.$ and $\left.0.70^{* *}\right)$, cob girth $\left(0.99 * *\right.$ and $\left.0.36^{* *}\right)$, number of kernel rows per $\operatorname{cob}\left(0.50^{* *}\right.$ and $\left.0.42^{* *}\right)$, number of kernels per $\operatorname{row}\left(3.20^{* *}\right.$ and $\left.3.08^{* *}\right), 100$ kernel weight $(4.01 * *$ and $1.78 * *)$ and kernel yield per plant $(265.69 * *$ and $144.23 * *)$, respectively. Further, the ratio of $\sigma^{2} \mathrm{gca} / \sigma^{2}$ scaless than one for all the characters studied also supported the greater importance of nonadditive genetic variance.

These results are in agreement with the findings of Amer et al., (2005), El- Hosary and El- Badway (2001), El- Hosary et al., (2000) and Sedham et al., (2007) in maize. It is evident from these results that heterosis breeding would be beneficial to utilize dominance gene effects for days to $50 \%$ tasseling, days to $50 \%$ silking, plant height, SPAD chlorophyll meter reading (SCMR), cob length $(\mathrm{cm})$, cob girth, number of kernel rows per cob, number of kernels per row, 100 kernel weight and kernel yield per plant.

\section{Mean performance, sca effects and Standard heterosis}

Significant sca effects and heterosis either positive or negative was recorded for all the characters studied. Percent heterosis varied from character to character and from cross to cross.. Heterosis over standard check is considered either to accept or reject a hybrid variety for commercial cultivation. Negative heterosis is desirable for days to $50 \%$ tasseling and silking for development of hybrids for early duration.

The extent of heterosis depends on the magnitude of non-additive gene action and genetic diversity of parental lines involved. The magnitude of $s c a$ effects and heterosis was found to be low for days to $50 \%$ tasseling and silking, SPAD chlorophyll meter reading, cob length, cob girth and number of kernel rows per cob compared to other characters. This may be due to the fact that greatest possible number of loci with dominant loci along with dominant alleles imparts vigour, size, yield, disease resistance and tolerance to environmental factors

Among the top 20 crosses, 12 and 15 crosses were found to be significantly superior during season when compared to standard check (DHM 117; BML 6x BML 7) for days to 50\% tasseling and 50\% silking. Four crosses viz., BML7 $\times$ DFTY $\quad\left(-0.884^{*}\right.$ and -0.981$)$; BML15×PDM1452(-1.926** and $\left.-1.690^{* *}\right)$; BML15×1474 (1.343** and 1.481**) and BML2X BML7(1.884** and -1.981) recorded significant negative sca effects and negative heterosis. 
Table.1 Anova for Combining Ability for Kernel Yield and Yield Components in Maize

\begin{tabular}{|c|c|c|c|c|c|c|}
\hline S. No & CHARACTER (S) & GCA (D.F=9) & SCA $(D . F=35)$ & $\sigma^{2}$ gca & $\sigma^{2}$ sca & $\sigma^{2}$ gca $/ \sigma^{2}$ sca \\
\hline 1. & Days to $50 \%$ tasseling & $7.66^{* *}$ & $4.11 * *$ & 0.90 & 3.61 & 0.25 \\
\hline 2. & Days to $50 \%$ silking & $10.83 * *$ & $5.99 * *$ & 1.27 & 5.33 & 0.24 \\
\hline 3. & Plant Height $(\mathrm{cm})$ & $335.63 * *$ & $296.56 * *$ & 35.62 & 290.90 & 0.12 \\
\hline 4. & SPAD Chlorophyll Meter Readings & $20.62 * *$ & $7.27 * *$ & 2.46 & 6.34 & 0.39 \\
\hline 5. & Cob length $(\mathrm{cm})$ & $5.94 * *$ & $1.88 * *$ & 0.70 & 1.48 & 0.47 \\
\hline 6. & Cob girth $(\mathrm{cm})$ & $3.01 * *$ & $1.01 * *$ & 0.36 & 0.99 & 0.40 \\
\hline 7. & Number of kernel rows per cob & $3.63 * *$ & $0.74 * *$ & 0.42 & 0.50 & 0.85 \\
\hline 8. & Number of kernels per row & $28.00 * *$ & $6.52 * *$ & 3.08 & 3.20 & 0.96 \\
\hline 9. & 100 kernel weight $(\mathrm{g})$ & $14.71 * *$ & $4.15^{* *}$ & 1.78 & 4.01 & 0.44 \\
\hline 10. & Kernel yield per plant $(\mathrm{g})$ & $1196.97 * *$ & $308.83 * *$ & 144.23 & 265.69 & 0.54 \\
\hline
\end{tabular}

*Significant at $5 \%$ level, **Significant at $1 \%$ level

Table.2 Mean per se performance, sca effects and standard heterosis for kernel yield and yield components in maize

\begin{tabular}{|c|c|c|c|c|c|c|c|c|c|c|c|c|c|c|c|c|}
\hline \multirow[t]{2}{*}{$\begin{array}{l}\text { S. } \\
\text { No }\end{array}$} & \multirow[t]{2}{*}{ HYBRID (s) } & \multicolumn{3}{|c|}{ Days to $50 \%$ Tasseling } & \multicolumn{3}{|c|}{ Days to $50 \%$ Silking } & \multicolumn{3}{|c|}{ Plant Height (cm) } & \multicolumn{3}{|c|}{$\begin{array}{c}\text { SPAD Chlorophyll Meter } \\
\text { Readings }\end{array}$} & \multicolumn{3}{|c|}{ Cob Length (cm) } \\
\hline & & Mean & $\begin{array}{l}s c a \\
\text { effects }\end{array}$ & SH & Mean & $\begin{array}{l}s c a \\
\text { effects }\end{array}$ & SH & Mean & $s c a$ effects & SH & Mean & sca effects & SH & Mean & $s c a$ effects & SH \\
\hline 1 & BML2×BML6 & 63.33 & $1.324 * *$ & 0.00 & 66.33 & $1.060^{*}$ & 0.51 & 191.20 & -1.232 & 4.37 & 49.33 & $-1.868 * *$ & -0.47 & 17.12 & -0.584 & -0.10 \\
\hline 2 & BML2×BML7 & 60.33 & $-1.884 * *$ & $-4.74 * *$ & 63.67 & $-1.981 * *$ & $-3.54 * *$ & 183.53 & -0.866 & 0.18 & 53.45 & $2.724 * *$ & $7.84 * *$ & 18.40 & $0.877^{* *}$ & $7.39 *$ \\
\hline 3 & BML2×Heypool & 62.00 & 0.449 & $-2.11 *$ & 67.00 & $1.977 * *$ & 1.52 & 190.00 & -1.07 & 3.71 & 53.52 & $0.867^{*}$ & $7.98 * *$ & 18.94 & 0.494 & $10.54 * *$ \\
\hline 4 & BML6×DFTY & 60.67 & $-1.009 * *$ & $-4.21 * *$ & 64.33 & -0.273 & $-2.53^{*}$ & 193.33 & -1.218 & 5.53 & 52.53 & -0.02 & $5.99 * *$ & 18.07 & -0.304 & 5.45 \\
\hline 5 & BML6×PDM1416 & 60.33 & $-1.301 * *$ & $-4.74 * *$ & 63.67 & $-0.940^{*}$ & $-3.54 * *$ & 183.33 & $-12.841^{* *}$ & 0.07 & 50.27 & 0.224 & 1.41 & 18.33 & $1.311^{* *}$ & $7.00 *$ \\
\hline 6 & BML6×PDM1452 & 61.33 & -0.093 & $-3.16 * *$ & 62.33 & $-2.023 * *$ & $-5.56 * *$ & 195.93 & -1.409 & $6.95^{*}$ & 49.95 & -0.736 & 0.77 & 17.18 & -0.449 & 0.29 \\
\hline 8 & BML7×DFTY & 61.00 & $-0.884 *$ & $-3.68 * *$ & 64.00 & $-0.981^{*}$ & $-3.03 * *$ & 178.00 & $-8.518^{*}$ & -2.84 & 53.69 & $1.605^{* *}$ & $8.31 * *$ & 19.12 & $0.927 * *$ & $11.60 * *$ \\
\hline 9 & BML7×PDM1452 & 62.33 & 0.699 & -1.58 & 65.67 & $0.935^{*}$ & -0.51 & 190.98 & 1.671 & 4.25 & 48.07 & $-2.147 * *$ & -3.03 & 17.30 & -0.154 & 0.97 \\
\hline 10 & BML15×Heypool & 62.33 & 0.449 & -1.58 & 66.33 & 0.769 & 0.51 & 191.67 & 6.716 & 4.62 & 50.40 & $-1.471 * *$ & 1.68 & 18.40 & 0.144 & $7.39 *$ \\
\hline 11 & BML15×PDM1452 & 59.67 & $-1.926 * *$ & $-5.79 * *$ & 63.67 & $-1.690 * *$ & $-3.54 * *$ & 173.29 & $-13.174 * *$ & -5.41 & 53.13 & $3.162 * *$ & $7.19 * *$ & 19.10 & $1.537 * *$ & $11.48 * *$ \\
\hline 12 & BML15×PDM1474 & 60.33 & $-1.343 * *$ & $-4.74 * *$ & 64.00 & $-1.481 * *$ & $-3.03 * *$ & 176.60 & $-8.030^{*}$ & -3.60 & 53.26 & $1.532 * *$ & $7.45 * *$ & 19.24 & $1.016^{* * *}$ & $12.32 * *$ \\
\hline 13 & DFTY ×Heypool & 62.00 & $0.782 *$ & $-2.11^{*}$ & 64.67 & 0.31 & $-2.02 *$ & 190.00 & -3.189 & 3.71 & 53.58 & -0.422 & $8.10 * *$ & 19.48 & 0.367 & $13.72 * *$ \\
\hline 14 & DFTY $\times$ PDM1452 & 60.67 & -0.259 & $-4.21 * *$ & 64.00 & -0.148 & $-3.03 * *$ & 201.47 & 6.768 & $9.97 * *$ & 53.38 & $1.280^{* *}$ & $7.70 * *$ & 19.53 & $1.109^{* *}$ & $14.01 * *$ \\
\hline 15 & DFTY×PDM1474 & 63.33 & $2.324 * *$ & 0.00 & 66.67 & $2.394 * *$ & 1.01 & 183.00 & $-9.868^{* *}$ & -0.11 & 52.83 & $-1.029^{*}$ & $6.59 * *$ & 18.83 & -0.254 & $9.92 * *$ \\
\hline 16 & Heypool×PDM1452 & 61.00 & 0.032 & $-3.68 * *$ & 64.33 & 0.227 & $-2.53^{*}$ & 199.93 & 3.953 & $9.13 * *$ & 53.60 & $1.462 * *$ & $8.14 * *$ & 17.41 & $-0.971 * *$ & 1.60 \\
\hline 18 & PDM1416×PDM1474 & 60.67 & -0.301 & $-4.21 * *$ & 64.00 & -0.273 & $-3.03 * *$ & 190.07 & -4.425 & 3.75 & 50.20 & $-1.151^{* *}$ & 1.28 & 17.18 & -0.559 & 0.27 \\
\hline 19 & PDM1428×PDM1474 & 62.00 & -0.634 & $-2.11 *$ & 64.67 & $-1.440 * *$ & $-2.02 *$ & 194.00 & -1.35 & 5.90 & 51.53 & $-1.125^{* *}$ & $3.97 *$ & 17.17 & -0.586 & 0.23 \\
\hline \multirow[t]{3}{*}{20} & PDM1452×PDM1474 & 62.00 & $1.241 * *$ & $-2.11^{*}$ & 65.67 & $1.644 * *$ & -0.51 & 206.55 & $10.894 * *$ & $12.75^{* *}$ & 52.93 & $0.939^{*}$ & $6.79 * *$ & 19.00 & $0.651 *$ & $10.89 * *$ \\
\hline & Standard Check & 63.33 & $0.95^{*}$ & 0.00 & 66.00 & 0.81 & $-6.60 * *$ & 183.20 & -5.95 & 0.00 & 49.57 & $-1.09 * *$ & 0.00 & 17.13 & -0.27 & 0.00 \\
\hline & Mean/SE $\left(\mathbf{g}_{\mathrm{i}}\right) / \mathbf{S . E}$ & 61.32 & 0.363 & -3.19 & 64.65 & 0.417 & -2.05 & 190.84 & 3.625 & 4.17 & 52.12 & 0.49 & 5.16 & 18.36 & 0.324 & 7.18 \\
\hline
\end{tabular}


Table.2 Contd.

\begin{tabular}{|c|c|c|c|c|c|c|c|c|c|c|c|c|c|c|c|c|c|}
\hline \multirow[t]{2}{*}{$\begin{array}{l}\text { S. } \\
\text { No }\end{array}$} & \multirow[t]{2}{*}{ HYBRID (S) } & \multicolumn{3}{|c|}{ Cob Girth (cm) } & \multicolumn{3}{|c|}{$\begin{array}{c}\text { Number of Kernel Rows per } \\
\text { Cob }\end{array}$} & \multicolumn{3}{|c|}{$\begin{array}{c}\text { Number of Kernels per } \\
\text { Row }\end{array}$} & \multicolumn{3}{|c|}{100 Kernel Wight (g) } & \multicolumn{3}{|c|}{ Kernel Yield per Plant (g) } & \multirow{2}{*}{$\begin{array}{c}\text { GCA } \\
\text { status } \\
\text { of } \\
\text { inbred } \\
\text { lines }\end{array}$} \\
\hline & & Mean & sca effects & SH & Mean & sca effects & SH & Mean & $\begin{array}{c}\text { sca } \\
\text { effects }\end{array}$ & SH & Mean & sca effects & SH & Mean & $\begin{array}{c}s c a \\
\text { effects }\end{array}$ & SH & \\
\hline 1 & BML2×BML6 & 14.52 & $-0.601 * *$ & 0.37 & 14.13 & -0.439 & 0.00 & 37.00 & -1.745 & -3.48 & 34.20 & 0.175 & $3.85 *$ & 143.85 & 5.801 & $19.45^{* *}$ & $\mathrm{G} \times \mathrm{P}$ \\
\hline 2 & BML2×BML7 & 16.00 & $1.065 * *$ & $10.60 * *$ & 14.37 & -0.01 & 1.65 & 41.00 & $1.880 *$ & 6.96 & 35.53 & $1.128 *$ & $7.89 * *$ & 142.73 & $8.850 * *$ & $18.52 * *$ & $\mathrm{G} \times \mathrm{P}$ \\
\hline 3 & BML2×Heypool & 16.08 & $0.558 * *$ & $11.18 * *$ & 15.67 & $0.532^{*}$ & $10.85 * *$ & 41.00 & 0.88 & 6.96 & 36.30 & 0.732 & $10.22 * *$ & 150.10 & $9.313 * *$ & $24.64 * *$ & $\mathrm{G} \times \mathrm{G}$ \\
\hline 4 & BML6×DFTY & 15.12 & -0.216 & $4.49 *$ & 14.90 & 0.051 & 5.42 & 40.00 & -0.12 & 4.35 & 34.87 & -0.115 & $5.87 * *$ & 138.90 & 0.221 & $15.34 * *$ & $P \times G$ \\
\hline 5 & BML6×PDM1416 & 16.04 & $1.424 * *$ & $10.85 * *$ & 14.83 & $0.776 * *$ & 4.95 & 39.00 & $1.921 *$ & 1.74 & 35.77 & $2.516^{* *}$ & $8.60 * *$ & 137.57 & $17.561 * *$ & $14.23 * *$ & $\mathrm{P} \times \mathrm{P}$ \\
\hline 6 & BML6×PDM1452 & 14.50 & $-0.558 * *$ & 0.23 & 14.27 & -0.258 & 0.94 & 38.67 & -0.287 & 0.87 & 33.20 & -0.732 & 0.81 & 140.25 & 4.601 & $16.46^{* *}$ & $\mathrm{P} \times \mathrm{P}$ \\
\hline 7 & BML6×PDM1474 & 16.08 & $0.661 * *$ & $11.15 * *$ & 15.67 & $0.560^{*}$ & $10.85 * *$ & 41.67 & 1.255 & $8.70 *$ & 36.25 & $0.868^{*}$ & $10.08 * *$ & 145.83 & 3.051 & $21.10 * *$ & $P \times G$ \\
\hline 8 & BML7×DFTY & 15.77 & $0.620 * *$ & $8.99 * *$ & 15.33 & $0.680 * *$ & $8.49 * *$ & 43.00 & $2.505^{* *}$ & $12.17 * *$ & 36.27 & $0.905^{*}$ & $10.12 * *$ & 146.70 & $11.520 * *$ & $21.82 * *$ & $\mathrm{P} \times \mathrm{G}$ \\
\hline 9 & BML7×PDM1452 & 14.80 & -0.072 & 2.30 & 14.07 & -0.262 & -0.47 & 39.00 & -0.329 & 1.74 & 35.00 & 0.688 & $6.28 * *$ & 136.07 & 4.166 & $12.99 * *$ & $\mathrm{P} \times \mathrm{P}$ \\
\hline 10 & BML15×Heypool & 15.70 & 0.335 & $8.53 * *$ & 15.10 & 0.179 & $6.84 *$ & 39.67 & -0.787 & 3.48 & 35.40 & -0.066 & $7.49 * *$ & 143.90 & $11.186^{* *}$ & $19.49 * *$ & $\mathrm{P} \times \mathrm{G}$ \\
\hline 11 & BML15×PDM1452 & 15.81 & $0.795 * *$ & $9.29 * *$ & 15.00 & $0.525^{*}$ & $6.13 *$ & 41.67 & $2.171 *$ & $8.70 *$ & 36.51 & $2.143 * *$ & $10.87 * *$ & 144.73 & $15.132 * *$ & $20.18 * *$ & $\mathrm{P} \times \mathrm{P}$ \\
\hline 12 & BML15×PDM1474 & 16.10 & $0.724 * *$ & $11.29 * *$ & 15.94 & $0.883 * *$ & $12.78 * *$ & 43.00 & $2.046^{*}$ & $12.17 * *$ & 37.90 & $2.077 * *$ & $15.08 * *$ & 143.77 & $6.782 *$ & $19.38 * *$ & $\mathrm{P} \times \mathrm{G}$ \\
\hline 13 & DFTY $\times$ Heypool & 15.92 & 0.179 & $10.02 * *$ & 15.23 & -0.179 & $7.78 * *$ & 41.67 & 0.171 & $8.70 *$ & 36.53 & 0.008 & $10.93 * *$ & 143.20 & 0.753 & $18.91 * *$ & $\mathrm{G} \times \mathrm{G}$ \\
\hline 14 & DFTY $\times$ PDM1452 & 16.18 & $0.795^{* *}$ & $11.87 * *$ & 15.57 & $0.601 *$ & $10.14 * *$ & 41.67 & 1.13 & $8.70 *$ & 36.40 & $0.971 * *$ & $10.53 * *$ & 143.40 & 4.065 & $19.08 * *$ & $\mathrm{G} \times \mathrm{P}$ \\
\hline 15 & DFTY $\times$ PDM1474 & 14.70 & $-1.049 * *$ & 1.61 & 14.40 & $-1.148 * *$ & 1.89 & 41.00 & -0.995 & 6.96 & 36.50 & -0.378 & $10.84^{* *}$ & 151.43 & 4.615 & $25.74 * *$ & $\mathrm{G} \times \mathrm{G}$ \\
\hline 16 & Heypool×PDM1452 & 14.72 & $-0.746 * *$ & 1.73 & 15.13 & 0.046 & $7.08 *$ & 39.67 & -0.662 & 3.48 & 35.90 & 0.425 & $9.01 * *$ & 138.80 & -0.368 & $15.26 * *$ & $\mathrm{G} \times \mathrm{P}$ \\
\hline 17 & Heypool×PDM1474 & 15.83 & 0.01 & $9.45^{* *}$ & 15.67 & -0.002 & $10.85 * *$ & 41.67 & -0.12 & $8.70 *$ & 36.30 & -0.628 & $10.22 * *$ & 145.77 & -0.784 & $21.04 * *$ & $\mathrm{G} \times \mathrm{G}$ \\
\hline 18 & PDM1416×PDM1474 & 14.27 & $-0.762 * *$ & -1.38 & 14.03 & $-0.723 * *$ & -0.71 & 41.33 & $2.380 *$ & $7.83 *$ & 34.07 & $-1.084 * *$ & 3.44 & 138.73 & $10.688 * *$ & $15.20 * *$ & $\mathrm{P} \times \mathrm{G}$ \\
\hline 19 & PDM1428×PDM1474 & 15.07 & 0.342 & $4.15 *$ & 14.37 & -0.248 & 1.65 & 37.33 & -1.329 & -2.61 & 34.67 & -0.327 & $5.26 * *$ & 140.85 & 7.994* & $16.96 * *$ & $P \times G$ \\
\hline \multirow[t]{3}{*}{20} & PDM1452×PDM1474 & 16.12 & $0.643 * *$ & $11.41 * *$ & 15.93 & $0.710 * *$ & $12.74 * *$ & 41.33 & 0.505 & $7.83 *$ & 36.61 & $0.781 *$ & $11.17 * *$ & 142.40 & -1.039 & $18.25 * *$ & $\mathrm{P} \times \mathrm{G}$ \\
\hline & Standard Check & 14.47 & -0.35 & 0.00 & 14.13 & -0.08 & 0.00 & 38.33 & -0.58 & 0.00 & 32.93 & $-0.93 *$ & 0.00 & 120.43 & $-10.82 * *$ & 0.00 & \\
\hline & Mean/SE $\left(g_{i}\right) / S . E$ & 15.47 & 0.18 & 6.91 & 14.98 & 0.256 & 5.99 & 40.52 & 0.929 & 5.70 & 35.71 & 0.361 & 8.43 & 142.95 & 3.345 & 18.70 & \\
\hline
\end{tabular}

*Significant at $5 \%$ level, **Significant at $1 \%$ level 
Out of 20 top yielding crosses eight have recorded significant negative sca effects while four have recorded positive sca effects. Sixteen crosses recorded significant negative heterosis, while four crosses recorded significant positive heterosis for days to $50 \%$ tasseling.

Two cross combinations DFTY $\times$ PDM1474 (1.50 and -3.07) and PDM1452×PDM 1472 (1.27 and -2.85) registered significant positive sca effects but significant negative heterosis, respectively. Eight crossesviz., BML2×PDM 1474, BML6×PDM 1452, BML15×Heypool, DFTY $\times$ Heypool, $\quad$ DFTY $\times$ PDM1428, DFTY $\times$ PDM1452, $\quad$ PDM1416 $\times$ PDM1474 registered non-significant sca effects and significant negative standard heterosis which is in desirable direction. For days to $50 \%$ silking 15of the top 20 crosses were found significantly early to silking over the standard check. Six crosses recorded significant negative sca effects while five have recorded significant positive sca effects. Fourteen crosses have recorded significant negative heterosis over standard check for days to $50 \%$ silking.

The cross combinations BML6×PDM1474, DFTY $\times$ PDM1452 and PDM1452×PDM1474 recorded significant higher mean value over standard check for plant height during rabi. Two crosses BML6×PDM1474 and PDM1452×PDM1474 recorded positive sca effects for plant height while five crosses viz., BML6 x PDM 1416,BML 7x DFTY, BML 15x PDM 1452, BML 15x PDM 1474 and DFTYXPDM 1474 have recorded significant negative sca effects. Five crosses BML6xPDM1474, DFTYx PDM 1452, HEYPOOLX PDM1452, HEYPOOLX PDM 1474 and PDM 1452x PDM 1474 recorded significant positive heterosis for plant height over standard check. Two crosses viz., BML6x PDM1474, PDM 1452x PDM1474 recorded significant positive values for both sca and heterosis. Of the top 20 crosses, 13 crosses have registered high mean performance over the standard check during Rabi for SPAD chlorophyll meter readings. Nine crosses registered significant positive sca effects while six have recorded significant negative sca effects. A total of 14 crosses have recorded positive and significant heterosis over check. The crosses viz., BML2 x BML7, BML2xHEYPOOL, BML6x PDM1474, BML7x DFTY, BML15X PDM1452, BML15X PDM1474, DFTYx PDM1452 and PDM 1452 x PDM 1474 recorded significant positive heterosis for this trait.

Fourteen cross combinations recorded significantly higher mean values over standard check for cob length during Rabi. Seven crosses recorded significant positive sca effects while two have recorded significant negative sca effects. Ten crosses have recorded significant positive heterosis. Nine cross combinations recorded significant positive sca effects and heterosis for cob length during the season.

High mean, positive significant sca effects and significant positive heterosis was observed in BML2 $\times$ BML7, BML2 $x$ HEYPOOL, BML6×PDM1416, BML6×PDM1474, BML7 $\times$ DFTY, BML15 $\times$ PDM1452, BML15× PDM 1474, DFTY $\times$ PDM1452 and PDM1452 xPDM 1474 for cob girth over the standard check during Rabi. A total of nine crosses showed high mean performance and positive significant sca effects while 14 crosses have recorded significant positive heterosis.

Eleven crosses registered significant higher mean values for number of kernel rows per cob. The cross combinations viz., BML2×HEYPOOL, BML6×PDM1474, BML 7x DFTY BML15x PDM 1452, BML15x PDM 1474, PDM 1452x PDM 1474, DFTY $x$ PDM 1452 registered high mean, significant positive $s c a$ effects and heterosis during Rabi 
over the standard check. Four crosses BML2×Heypool, BML2×PDM1474, DFTY× Heypool and Heypool $\times$ PDM1474 exhibited high mean performance, non-significant sca effects but significant positive heterosis. A total of 10 and11 crosses have recorded significant positive sca effects and heterosis, respectively.

Fornumber of kernels per row thirteen crosses have recorded higher mean values during Rabi over the standard check. Thirteen crosses for positive significant sca effects and nine crosses for significant positive heterosis were found superior over standard check. The cross combinations viz., $\quad$ BML7 $\times$ DFTY, BML15×PDM1452 and BML15×PDM1474, DFTYX PDM 1474 recorded significant positive sca effects and heterosis over standard check for number of kernels per row.

Six crosses viz., BML2×BML7, BML6× PDM11416, BML6×PDM1474, BML7x DFTY, BML15×PDM1452, BML15 $\times$ PDM1474, BML1452×PDM1474, DFTY $x$ PDM 1452 of the top 20 exhibited high mean performance, significant positive sca effects and positive significant heterosis during over standard check. A total of nine crosses and 18 crosses recorded significant positive heterosis and $s c a$ effects were recorded for 100 kernel weight during Rabi.

Among the top yielding crosses, nine exhibited significant positive sca effects while all crosses showed significant positive heterosis for kernel yield per plant when compared with standard check. High mean, high positive and significant heterosis and significant positive $s c a$ effects were registered byBML $2 \times$ Heypool, BML2 $\times$ BML7, and BML6×PDM1416, BML7x DFTY, BML15× Heypool, BML15×PDM 1452, BML15×PDM 1474, PDM 1428×PDM 1452 and PDM 1452x PDM 1474 cross combinations for grain yield during Rabi. High heterosis for yield of maize was also reported by El- Badway et al., (2000), Shafey et al., (2004), Kanta et al., (2005), Alamnic et al., (2006), El-Hosary et al., (2006), Hefney (2007) and Sedhom et al., (2007).

The magnitude and extent of heterosis in desired direction is of importance in deciding economical importance of heterosis. High heterosis for grain yield had contribution from high heterosis of yield components (Moneam et al., 2008). In present study, three crosses viz; BML7×DFTY, BML15×PDM1452 and BML15×PDM1474 were heterotic for kernel yield over standard check. In addition these cross combinations also recorded, high per seperformance, significant positive heterosis and sca effects for most of the yield contributing characters viz., days to $50 \%$ tasseling, days to $50 \%$ silking, SPAD chlorophyll meter reading, cob length, cob girth, number of kernel rows per cob, number of kernels per row and 100 kernel weight. These outstanding cross combinations involved good $\times$ poor or poor $\times$ poor general combiners as parents. Hence, these three cross combinations can be put for immediate commercial cultivation after extensive testing for their performance under different locations and environments.

The crosses with yielding potential involving good $\times$ good general combiners viz., DFTY $\times$ PDM1474, DFTY $\times$ Heypool and BML2 $\times$ Heypool and which recorded significant positive heterosis over standard check significant or non-significant $s c a$ effects and low magnitude of heterosis for all other yield contributing characters may be handled through population improvement programme so as to accumulate favourable genes and to develop improved inbred lines and hybrids. The cross combinations viz., BML2×BML7, BML2 $\times$ PDM1428, DFTY $\times$ PDM1452, BML6 $\times$ PDM1452, BML6×PDM1474, BML15× Heypool, DFTY $\times$ PDM 1428, Heypool $\times$ 
PDM1452, PDM1416×PDM1474, PDM1428 $\times$ PDM1474 and PDM1452 $\times$ PDM1474 involving either good $\times$ poor, poor $\times$ good, poor $\times$ poor combinations possessing high mean performance, significant positive heterosis and significant or non-significant sca effects may be handled by heterosis breeding to exploit hybrid vigour and develop high yielding hybrids.

Additive and non-additive type of genetic variance were found to be governing days to $50 \%$ tasseling, days to 50\% silking, plant height, SPAD chlorophyll meter reading (SCMR), cob length $(\mathrm{cm})$, cob girth $(\mathrm{cm})$, number of kernel rows per cob, number of kernels per row, 100 kernel weight and kernel yield per plantwith predominance of nonadditive gene action. The magnitude of standard heterosis was found to be low for developmental characters, cob length, cob girth, number of kernel rows per cob compared to number of kernels per row, 100 kernel weight, plant height and kernel yield per plant. The crosses viz., BML7×PDM1474, BML15×PDM1452 and BML15×PDM1474 which involved poorxgood combinations coupled significant positive sca effects and positive heterosis for most of the yield contributing characters are considered as outstanding crosses which can be recommended for commercial cultivation after extensive testing under different environments and locations. Four crosses viz; BML 2x Heypool, DFTY x Heypool, DFTY x PDM 1474 and Heypool x PDM 1474 which involved high $\mathrm{x}$ high general combiners with high mean either significant or non-significant sca effects and significant positive heterosismay be handled through recurrent selection programme so as to accumulate desirable alleles and to isolate improved hybrids/inbred lines. All other cross combinations involving good $\times$ poor, poorxgood or poorxpoor may be handled through heterosis breeding to exploit hybrid vigour and to develop improved hybrids.

\section{References}

Akbar, M., Saleem, M., Azhar, F. M., Ashraf, M. Y and Ahmad, R. 2008. Combining ability analysis in maize under normal and high temperature conditions. Journal of Agricultural Research. 46(1): 261-277.

Amer, E. A. 2005. Estimates of combining ability using diallel crosses among eight new maize inbred lines. J. Agric. Res. Tanta. Univ. 31:67-73.

Amiruzzaman, M., Islam, M. A., Hassan, L., Monjurul Kadir and Rohman, M. M. 2013. Heterosis and combining ability in a diallel among elite inbred lines of maize (Zea mays L.) Emirates Journal of Food and Agriculture., 25(2): 132137.

Azad M. A. K., Biswas B. K., Alam N and Alam S. K. 2014. Combining Ability and Heterosis for Some Quantitative Traits in Experimental Maize Hybrids. Journal of Plant Breeding and Seed Science.70: 41-54.

Dar, Z. A., Lone, A. A., Ali G., Abidi, I., Lone, R. A., Gulzar, S., Abrar Yasin., Yusra Ali and Yousuf, N. 2017. Gene action studies in early maturing maize (Zea mays L.) inbred lines. Archives of Agriculture and Environmental Science. 2 (2): 68-71.

Debnath, S. V and Sarkar, K. R. 1990. Combining ability analysis of grain and some of its attributes in maize (Zea mays L.). Indian Journal of Genetics and Plant Breeding. 50: 5761.

El- Badway, El-Badawy, M. El. M. 2006. Genetic analysis of diallel crosses in maize (Zea mays L.). J. Agric Res. Benha Univ. 44:911-922.

El-Badawy, M. El. M. 2013. Heterosis and Combining Ability in Maize using Diallel Crosses among Seven New Inbred Lines. Asian Journal of Crop 
Science. 5(1): 1-13.

Griffing, B. 1956. Concept of general and specific combining ability in relation to diallel crossing systems. Australian Journal of Biological Sciences. 9: 46393.

Hayes, H. K., Immer, F. R and Smith, D. C. 1955. Methods of plant breeding. second edition. McGraw Hill co. New York, U.S. A.

Hefney, M. 2010. Genetic control of flowering traits, yield and its components in maize (Zea mays L.) at different sampling dates. Asian J. Crop Sci. 3:116-117.

Iqbal, A. M., Nehui, F. A., Wani, S. A., Qadir, $\mathrm{R}$ and Dar, Z. A. 2007. Combining ability analysis for yield and yield related traits in maize (Zea mays L.). Intl. J. Plant Breed. Genet. 1:101-105.

Iqbal, M. K., Khan, H., Rahman, I. H., Khalil, H., Sher and Bakht, J. 2010. Heterosis for morphological traits in subtropical maize (Zea mays L.). Maydica.55: 4148.

Jinks, J. 1954. The analysis of continuous variation in a diallel cross of Nicotianarustica varieties. Genetics. 39(6): 767.

Mohammad AbulKalam Azad., Bhabendra Kumar Biswas., NazmulAlam and Sheikh Shamimul Alam. 2014. Combining Ability and Heterosis for Some Quantitative Traits in Experimental Maize Hybrids. Plant Breeding and Seed Science. 70.

Praveen Kumar, G., Prashanth Y., Narsimha
Reddy, V., Sudheer Kumar S and Venkateshwara Rao, P. 2014. Heterosis for Grain yield and its Component traits in Maize (Zea mays L.). Intl. J. Pure \& Appl. Biosci. 2(1): 106-111.

Rajesh, V., Sudheer Kumar, S., Narsimha Reddy, V and Siva Sankar, A. 2018. Combining Ability and Genetic Action Studies for Yield and Its Related Traits in Maize (Zea mays L.). Intl. J. Curr. Microbiol. and Appl. Sci.7(6): 26452652.

Rumana Khan and Dubey R. B. 2015. Combining ability analysis for Nutritional Quality and Yield in Maize (Zea mays L.). The Bioscan. 10(2): 785-788.

Sandesh, G. M., Karthikeyan, A., Kavithamani, D., Thangaraj, K., Ganesan, K. N., Ravikesavan, R and Senthil, N. 2018. Heterosis and combining ability studies for yield and its component traits in Maize (Zea mays L.). Eltr.J. of Plant Breed. 9(3): 1012-1023.

Sedhom, A. S., M. El-Badway, M. E., Morsy, A. M and El-Hosary, A. A. A. 2007. Diallel analysis and relationship between molecular polymorphism and yellow maize hybrid performance. $J$. Agric. Sci. Benhauniv. 45:1-20.

Turner, J. K. 1953. A study of heterosis in upland cotton -II. Combining ability and inbreeding effects. Agron. J. 45:487-490.

\section{How to cite this article:}

Sabitha, N., D. Mohan Reddy, D. Lokanath Reddy, M. Hemanth Kumar, P. Sudhakar and Ravindra Reddy, B. 2021. Combining Ability and Heterosis for Kernel Yield and Yield Attributes in Maize (Zea mays L.). Int.J.Curr.Microbiol.App.Sci. 10(06): 198-206. doi: https://doi.org/10.20546/ijcmas.2021.1006.021 